\title{
昡pubvet
}

https://doi.org/10.31533/pubvet.v14n9a650.1-7

\section{Aspectos epidemiológicos, clínicos e patológicos da babesiose bovina}

\author{
Lucas de Souza Quevedo $^{1 *}$, Pedro de Souza Quevedo 29 \\ ${ }^{1}$ Laboratório de Patologia Animal, Departamento de Medicina Veterinária, Centro de Ciências Agroveterinárias (CAV), Universidade do \\ Estado de Santa Catarina (UDESC), Av. Luiz de Camões 2090, Bairro Conta Dinheiro, Lages, SC, Brasil. \\ ${ }^{2}$ Centro de Diagnóstico e Estudos em Parasitologia Animal, Instituto de Estudos do Trópico Úmido (IETU), Universidade Federal do Sul e \\ Sudeste do Pará (UNIFESSPA), Rua Santos Dumont S/N, Bairro Jardim Universitário, Xinguara, PA, Brasil. \\ *Autor para correspondência, E-mail: souzaquevedo@gmail.com
}

Resumo. A babesiose bovina é uma enfermidade com curso febril e hemolítico, que juntamente com a anaplasmose formam o chamado complexo da tristeza parasitária bovina (TPB). No Brasil, a babesiose é transmitida aos bovinos pelo carrapato Ripicephalus (Boophilus) microplus, de ocorrência em praticamente todo o território nacional. Os impactos econômicos da babesiose compreendem a queda de rendimento dos animais, com redução da produção de leite, emagrecimento, aborto e mortalidade. A infecção pelos protozoários Babesia bovis e Babesia bigemina provoca hemólise intravascular de efeitos potencialmente fatais. Em infecções por $B$. bovis pode ser observado um quadro neurológico grave em decorrência da congestão dos capilares encefálicos, com hemácias parasitadas. A consequente hipóxia no sistema nervoso central culmina com manifestações de incoordenação motora, andar cambaleante, agressividade e movimentos de pedalagem. Casos de babesiose por B. bigemina tem por característica, além da febre e prostração, a hemoglobinúria. Conhecer aspectos da epidemiologia, sinais clínicos, conduta terapêutica e formas de controle e profilaxia referentes a babesiose bovina é fundamental aos médicos veterinários de campo. Diante da grande importância da babesiose bovina e seus efeitos deletérios à pecuária, a presente revisão aborda aspectos sobre epidemiologia, ciclo biológico dos protozoários, sinais clínicos, lesões post mortem e subsídios ao diagnóstico da enfermidade.

Palavras chave: Babesia bovis, Babesia bigemina, Tristeza parasitária bovina

\section{Epidemiological, clinical and pathological findings of bovine babesiosis}

\begin{abstract}
Bovine babesiosis is a disease with a febrile and hemolytic course, which together with anaplasmosis form the so-called bovine parasitic sadness complex (TPB). In Brazil, babesiosis is transmitted to cattle by the tick Ripicephalus (Boophilus) microplus, which occurs in practically the entire national territory. The economic impacts of babesiosis include the drop in animal performance, with reduced milk production, weight loss, abortion and mortality. Infection with the Babesia bovis and Babesia bigemina protozoa causes intravascular hemolysis with potentially fatal effects. In infections by B. bovis, a severe neurological condition can be observed due to congestion of the brain capillaries, with parasitized red blood cells. The consequent hypoxia in the central nervous system culminates in manifestations of motor incoordination, aggressiveness and pedaling movements. In addition to fever and prostration, babesiosis caused by B. bigemina has hemoglobinuria. Knowing aspects of epidemiology, clinical signs, therapeutic conduct and forms of control and prophylaxis related to bovine babesiosis is essential for field veterinarians. In view of the great importance of bovine babesiosis and its deleterious effects on livestock, this review addresses aspects about epidemiology, the biological cycle of protozoa, clinical signs, post-mortem injuries and subsidies for the diagnosis of the disease.
\end{abstract}

Keywords: Babesia bovis, Babesia bigemina, Cattle tick fever 


\section{Introdução}

A tristeza parasitária bovina (TPB) é responsável por causar grandes prejuízos à pecuária (Almeida et al., 2006). Trata-se de um complexo formado por babesiose e analasmose. Por este motivo, os termos por vezes se sobrepõem com tristeza parasitária bovina. Animais clinicamente acometidos pela babesiose apresentam anemia hemolítica associada à febre e com intensidade variando de acordo com o(s) agente(s) etiológico(s), quantidade de formas infectantes e o status imunológico (Taylor et al., 2017). A babesiose ocorre em várias regiões do Brasil, como sertão da Paraíba (Costa et al., 2018), região sudeste (Gonçalves et al., 2011) e Sul (Almeida et al., 2006). A enfermidade pode acometer desde neonatos até bovinos adulto sem experiência imunológica para os agentes da TPB (Almeida et al., 2006; Krolow, 2002; Santarosa et al., 2013). Surtos de babesiose ocorrem devido a inoculação de grandes quantidades de formas infectantes de Babesia spp., fatores imunossupressores como carência nutricional, ou a perda da resistência imunológica devido longo período sem contato com o protozoário (Barros et al., 2006; Farias, 1995).

\section{Revisão}

A babesiose bovina é uma enfermidade causada pelos protozoários Babesia bovis e Babesia bigemina. Ambas as espécies são transmitidas pelo carrapato do boi Ripicephalus (Boophilus) microplus e, juntamente com a anaplasmose, determinada por Anaplasma marginale, formam o complexo Tristeza Parasitária Bovina (TPB) (Almeida \& Andreotti, 2013; Farias, 2007).

As babésias possuem como característica principal a infecção e multiplicação em eritrócitos. É reconhecido que B. bovis é a espécie mais patogênica aos bovinos (Barros et al., 2006; Taylor et al., 2017). As consequências ao hospedeiro se devem a hipóxia e anemia, desencadeadas devido ao consumo de hemoglobina durante sua permanecia na célula e, ruptura de membrana plasmática após a exocitose (Farias, 1995).

Cumpre salientar que o grau de anemia é muitas vezes desproporcional a parasitemia, principalmente em infecções por B. bovis. Por vezes, uma redução drástica do hematócrito pode ser associada com infecções de menos de 1\% dos eritrócitos (Taylor et al., 2017).

Podem ser acometidos bovinos, bubalinos e cervídeos, em todas as idades, entretanto cada um destes agentes possui características epidemiológicas distintas (

Os protozoários B. bovis e B. bigemina, estão presentes em países da África, América Central e do Sul, Ásia e sul da Europa além da Austrália. Na América do Norte não é citada a presença de B. bovis, no entanto, B. bigemina está presente neste continente (Taylor et al., 2017).

Ainda que os agentes etiológicos da babesiose tenham ampla distribuição, existem diferentes situações epidemiológicas para TPB no Brasil. Estes cenários descritos compreendem áreas livres, áreas de instabilidade (epidêmicas) e regiões de estabilidade enzoótica (endêmicas) (Almeida \& Andreotti, $\underline{2013}$.

As áreas consideradas livres no Brasil são onde não ocorrem infestações por carrapatos, como as regiões do extremo sul do Rio Grande do Sul, nos municípios de Santa Vitória do Palmar e Chuí (Almeida et al., 2006). Em tais cenários epidemiológicos podem ocorrer surtos acidentais, quando há entrada de carrapatos em períodos favoráveis ao ciclo biológico do ectoparasito (Farias, 2007).

Nas demais localidades do Rio Grande do Sul, os períodos em que mais ocorre a TPB estão compreendidos entre as estações do verão e o outono, $42 \%$ dos casos em abril e maio (Almeida et al., 2006). Isso ocorre por tratar-se do período de maior infestação de carrapato, responsável pela transmissão dos agentes etiológicos da TPB, favorecidos pelas condições climáticas sazonais (Krolow, 2002).

Por estas características as áreas externas aos municípios de Santa Vitória do Palmar e Chuí são consideradas áreas de instabilidade ou epidêmicas (Farias \& Lemos, 2007).

Entre os fatores que credenciam uma área como epidêmica à ocorrência de TPB estão a umidade $\mathrm{e}$ temperatura, que determinam menor tempo de infestação por carrapatos (Almeida et al., 2006). Os 
carrapatos inoculam esporozoítos, a forma infectante das babésias, nos bovinos desencadeando a produção de anticorpos contra o protozoário (Farias, 1995; Taylor et al., 2017).

Quando a população de carrapatos decresce por intermédio de tratamentos supressivos ou condições ambientais adversas ao ciclo evolutivo, o contato dos bovinos com o protozoário não acontece. Consequentemente há um decréscimo nos níveis de anticorpos contra Babesia spp. (Farias, 1995; Almeida \& Andreotti, 2013).

Dessa forma, os animais destas regiões ficam susceptíveis, além disso, para ser considerada uma região epidêmica é estabelecido que o percentual de animais que possuam sorologia positiva para Babesia sp. deva ser de 15 - 80\% (Farias, 2007; Farias, 1995).

Áreas endêmicas são todas as outras regiões do Brasil que possuem infestação por carrapatos $R$. microplus durante todo o ano. A exposição ao agente faz o animal estimular seu sistema imune, entretanto alguns produtores fazem o combate ao vetor isso leva a diminuição desta imunidade e a ocorrência de casos isolados ou até mesmo surtos (Almeida \& Andreotti, 2013).

Quanto a origem dos animais, há uma reconhecida susceptibilidade maior em bovinos Bos taurus taurus do que Bos taurus indicus (Costa et al., 2018; Rodrigues et al., 2005). Cumpre salientar que a origem do carrapato do boi $R$. microplus remete a Índia e Ilha de Java na Ásia (Matias et al., 2013). As raças que evoluíram na presença de desafios constantes pelo carrapato apresentam maior resistência ao ectoparasito. Dessa forma é reconhecido que zebuínos comumente estabelecem uma relação estável com carrapatos (Siqueira et al., 2013).

A faixa etária de animais acometidos varia de 6 meses e 8 anos de idade (Almeida et al., 2006; Rodrigues et al., 2005). E os animais que não se infectam até os nove meses de idade não montam respostas imunológicas efetivas, tornando-se mais vulneráveis (Almeida \& Andreotti, 2013). Cabe salientar que antes da primo-infecção os animais são protegidos por anticorpos colostrais, que contra $B$. bovis permanecem em titulações apreciáveis até no máximo 90 dias e, conta B. bigemina, 60 dias de vida (Costa et al., 2018; Farias, 1995).

Animais entre 7 e 10 meses de idade apresentam imunidade não específica conta $B$. bovis e $B$. bigemina. Por tanto a imunidade passiva, adquirida via colostro dura cerca de dois meses e deve preceder o contato com os agentes etiológicos da babesiose, para garantir uma titulação protetiva de anticorpos contra B. bovis e B. bigemina (Farias \& Lemos, 2007).

Via de regra animais infectados com 6 a 9 meses de idade não apresentam sinais clínicos e desenvolvem imunidade duradoura (Rodrigues et al., 2005).

Os agentes etiológicos da TPB, B. bovis, B. bigemina e A. marginale são inoculados por carrapatos do boi, $R$. microplus. A anaplasmose ainda pode ser transmitida de forma iatrogênica (instrumentos de castração e vacinação), transplacentária ou por moscas hematófagas, mutucas e culicídios (Kessler, 2001; Taylor et al., 2017).

O período de incubação de Babesia sp. é de 7 - 10 dias e de Anaplasma marginale é geralmente maior que 20 dias. O protozoário $B$. bovis começa a ser transmitido ao bovino já no primeiro dia de parasitismo por larvas do carrapato R. microplus (Farias, 1995). Cabe salientar que existe a possibilidade da transmissão congênita de B. bovis, com consequente geração de natimortos ou mortalidade de animais antes do primeiro mês de vida (Bracarense et al., 2001; Oliveira et al., 2018).

A inoculação de esporozoítos de B. bigemina ao bovinos ocorre apenas após 8 dias de parasitismo, quando o carrapato atinge o estágio ninfal (Almeida \& Andreotti, 2013).

Então animais sem imunidade podem desenvolver babesiose por B. bovis $7-10$ dias após serem introduzidos em um novo campo infestado por carrapatos, babesiose por B. bigemina entre $15-20$ dias e se manifestarem sinais de TPB em um período superior a 20 dias, poderá ser indicativo de Anaplasmose (Almeida \& Andreotti, 2013; Farias \& Lemos, 2007).

Em condições de campo esses períodos nem sempre respeitam os dados constatados em condições controladas. Por vezes é possível observar animais infectados por um, dois ou até mesmo os três agentes etiológicos da TPB (Almeida et al., 2006; Costa et al., 2018). Nesse contexto, conhecimentos 
relacionados ao ciclo biológico dos agentes etiológicos e condições epidemiológicas devem ser aliados a patogenia e sinais clínicos (Tabela 1) para o estabelecimento do diagnóstico da TPB.

Tabela 1. Agentes etiológicos da TPB com respectiva patogenia e sinais clínicos.

\begin{tabular}{lcc}
\hline Agente & Patogenia & Sinais Clínicos \\
\hline $\begin{array}{l}\text { Babesia } \\
\text { bovis }\end{array}$ & $\begin{array}{c}\text { Mais patogênica, o grau de anemia é desproporcional a } \\
\text { parasitemia, é associada a deposição de hemácia a } \\
\text { pequenos capilares que no cérebro causa anoxia e lesão } \\
\text { tissular, pode ainda causar estase circulatória, choque e } \\
\text { coagulação intravascular. }\end{array}$ & $\begin{array}{c}\text { Hemoglobinúria, temperatura superior a 41 }{ }^{\circ} \text { C, } \\
\text { incordenação motora, andar cambaleante, } \\
\text { movimentos de pedalagem e agressividade. }\end{array}$ \\
$\begin{array}{l}\text { Babesia } \\
\text { bigemina }\end{array}$ & $\begin{array}{r}\text { Rápida multiplicação do parasito e destruição de } \\
\text { eritrócitos. Menor virulência. }\end{array}$ & $\begin{array}{c}\text { Apatia, orelhas caídas, debilidade, febre, anorexia, } \\
\text { emagrecimento, pelos arrepiados, taquicardia, } \\
\text { taquipneia, redução dos movimentos de ruminação, } \\
\text { redução ou suspensão da lactação, anemia e } \\
\text { hemoglobinúria. }\end{array}$ \\
\hline $\begin{array}{l}\text { Anaplasma } \\
\text { marginale }\end{array}$ & $\begin{array}{c}\text { Período de incubação de 4 semanas, ocorre febre } \\
\text { parasitemia e com a evolução se torna mais grave, com } \\
\text { até 70\% dos eritrócitos destruídos. }\end{array}$ & $\begin{array}{c}\text { Apatia, orelhas caídas, debilidade, febre, anorexia, } \\
\text { emagrecimento, pelos arrepiados, taquicardia, } \\
\text { taquipneia, redução dos movimentos de ruminação, } \\
\text { redução ou suspensão da lactação e icterícia. }\end{array}$ \\
\hline
\end{tabular}

Fonte: Adaptado de (Almeida \& Andreotti, 2013; Barros et al., 2006; Farias, 1995; Taylor et al., 2017).

Entre as alterações macroscópicas da babesiose estão a esplenomegalia, hepatomegalia, hemoglobinúria, icterícia, hemorragias cardíacas e bile verde-escura espessa (Almeida et al., 2006; Barros et al., 2006; Oliveira et al., 2018; Taylor et al., 2017). Como alteração patognomônica em casos de TPB, causados por B. bovis, é contatada uma pronunciada congestão do córtex encefálico, conferindo-lhe um aspecto de coloração "róseo-cereja" (Barros et al., 2006; Oliveira et al., 2018).

Como achados adicionais em animais com babesiose podem ser descritos a carcaça pálida, pulmões edematosos, saco pericárdico com presença de fluido sorossanguinolento, mucosas do abomaso e intestinal ictéricas com sinais de hemorragias subserosas e bexiga distendida com urina escura castanhoavermelhada (Barros et al., 2006; Taylor et al., 2017).

Os achados macroscópicos e os achados histopatológicos são semelhantes aos de doenças que causam hemólise intravascular e anemia, com sinusóides hepáticos distendidos e repletos de sangue, degeneração de hepatócitos, canalículos biliares distendidos contendo bile, linfonodos com maior número de macrófagos e hemácias no seu interior, congestão capilar do cérebro com edema perivascular e pequenas hemorragias, e necrose e congestão vascular nos rins (Barros et al., 2006; Oliveira et al., $\underline{2018})$

Porém para a determinação do diagnóstico definitivo não se faz necessário a avaliação histopatológica. É possível a confirmação a partir de lesões macroscópicas juntamente com a realização da técnica denominada clapp (impressão do órgão na lâmina), do córtex encefálico seguido da coloração pela técnica de Giemsa (Farias, 1995).

Durante a avaliação em microscópio e auxílio de óleo de imersão é possível visualizar pequenas corpos arredondados ou corpos pareados em forma de pêra unidos em ângulo obtuso dentro de hemácias, estas estruturas possuem de $1-1,5 \mu \mathrm{m}$ (arredondados) e 1,5 - 2,4 $\mu \mathrm{m}$ (forma de pêra) (Farias, 1995; Santarosa et al., 2013; Taylor et al., 2017).

Outra alternativa de diagnóstico definitivo possível utilizada é Método Panótico rápido. Com esta coloração é possível observar capilares do córtex encefálico repletos por hemácias contendo estruturas basofílicas solitárias ou pareadas de aproximadamente $2 \mu \mathrm{m}$ de diâmetro (Antoniassi et al., 2009).

O protozoário B. bovis possui predileção por vasos de pequenos calibres, e B. bigemina é encontrado geralmente na circulação geral, diante disto fica evidente a eficiência na determinação do agente causador da doença em esfregaço de córtex encefálico (Barros et al., 2006; Rodrigues et al., 2005). Cumpre salientar que para um diagnóstico correto é necessário que não apenas fragmentos de encéfalo sejam submetidos ao laboratório referência do médico veterinário, mas que todos os procedimentos de uma necropsia sejam respeitados. 
Para o tratamento da doença são indicados fármacos conforme descritos a seguir na Tabela 2. As doses preconizadas visam o tratamento de babesiose por B. bovis, a mais patogênica (Barros et al., 2006). O imidocarb é o fármaco considerado de dupla ação, sua utilização deve ser acompanhada por cuidados específicos e uma medicação suporte com hepatoprotetor, soro glicosado, anti-histamínico, sombra, água fresca, alimento a disposição e sem muita movimentação (Farias \& Lemos, 2007; Farias, 1995).

Tabela 2. Tratamento da babesiose. Princípio ativo, dose e frequência de administração.

\begin{tabular}{llc}
\hline Princípio ativo & Dose & Frequência \\
\hline Diaceturato de diminazina & 3 a $5 \mathrm{mg} / \mathrm{kg}$ & $2-3$ aplicações a cada $24 \mathrm{~h}$ \\
Dipropionato de imidocarb & 1 a $3 \mathrm{mg} / \mathrm{kg}$ & $2-3$ aplicações a cada $24 \mathrm{~h}$ \\
\hline
\end{tabular}

Fonte: Adaptado de (Barros et al., 2006; Farias, 2007).

Para controle da TPB em para regiões livres de carrapatos como o município de Santa Vitória do Palmar é recomendado evitar a entrada do vetor ( $R$. microplus), e que animais oriundos destas regiões, antes de serem introduzidos a locais endêmicos sejam previamente imunizados (Farias \& Lemos, 2007).

Em regiões endêmicas é preconizado que animais jovens entrem em contato com carrapatos para criarem imunidade contra os agentes da TPB, caso esta infestação seja muito grande deve ser realizado o controle do ectoparasita. Podem ser realizados banhos estratégicos em áreas epidêmicas e endêmicas

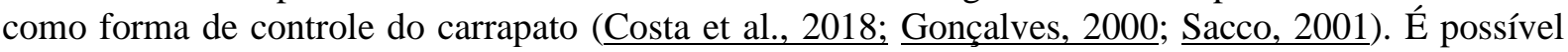
também a utilização da premunição, vacinação ou quimiprofilaxia (Sacco, 2001).

Premunição é uma técnica rudimentar e consiste em inocular sangue de um bovino infectado com os agentes da TPB em animais susceptíveis. Esse procedimento pode resultar em um processo de infecção clínica, categorizando a técnica como arriscada, aliado ao fato de possíveis transmissões de outros patógenos ( $\underline{\text { Sacco, 2001) }}$.

A maioria das vacinas contra a TPB consistem em cepas atenuada de B. bovis e B. bigemina somadas a $A$. centrale (menos patogênico que o A. marginale). Este inócuo é padronizado e o animal apresenta uma infecção subclínica, é uma medida mais segura, pois não apresenta sua forma virulenta normal (Sacco, 2001; Santos et al., 2013).

O método quimioprofilático consiste na aplicação de dipropionato de imidocarb aliado a infestação dos animais por carrapatos. Nesse método os animais apresentam uma queda gradual dos níveis sanguíneos do fármaco e aumento gradual do inoculo dos agentes da babesiose, possibilitando o desenvolvimento dos mecanismos de imunidade (acco, 2001; Barros et al., 2006).

Deve-se levar em consideração como diagnóstico diferencial da babesiose, doenças que desenvolvam quadros de anemia, icterícia e hemoglobinúria como leptospirose, hemoglobinúria bacilar, intoxicações por Cobre, Senna occidentalis, samambaia e Brachiaria spp (Farias \& Lemos, 2007) Doenças que cursam com quadros neurológicos como raiva e polioncefalomalácia (Rodrigues et al., 2005). Estas doenças podem ser eliminadas do diagnóstico pela epidemiologia que cada uma apresenta, achados clínicos e patológicos, entretanto a congestão encefálica vermelho-cereja ou o esfregaço do córtex são suficientes para fechar o diagnóstico definitivo de Babesiose por B. bovis (Antoniassi et al., 2009).

\section{Considerações finais}

Conhecer os sinais clínicos, condições epidemiológicas, lesões macroscópicas e microscópicas, diagnóstico e tratamento dos agentes da babesiose bovina são preceitos inerentes à atuação do médico veterinário de campo. Por sua vez, devido as consequências dramáticas ao organismo dos bovinos, é imperativo que pecuaristas compreendam a situação epidemiológica quanto a babesiose em que estão inseridos. Dessa forma é possível a adoção de medidas de controle e profilaxia dessa importante causa de perdas ao setor.

\section{Referências bibliográficas}

Almeida, M. B., Tortelli, F. P., Riet-Correa, B., Ferreira, J. L. M., Soares, M. P., Farias, N. A. R., RietCorrea, F., \& Schild, A. L. (2006). Tristeza parasitária bovina na região sul do Rio Grande do Sul: 
estudo retrospectivo de 1978-2005. Pesquisa Veterinária Brasileira, 26(4), 237-242. https://doi.org/10.1590/S0100-736X2006000400008

Almeida, R. F. C., \& Andreotti, R. (2013). Principais doenças transmitidas por carrapatos no Brasil, In: Carrapatos no Brasil. In W. W. - Brasília (Ed.), Embrapa, Brasılia.

Antoniassi, N. A. B., Corrêa, A. M. R., Santos, A. S., Pavarini, S. P., Sonne, L., Bandarra, P. M., \& Driemeier, D. (2009). Surto de babesiose cerebral em bovinos no Estado do Rio Grande do Sul. Ciência Rural, 39(3), 933-936. https://doi.org/10.1590/S0103-84782009000300049

Barros, C. S. L., Driemeier, D., Dutra, I. S., \& Lemos, R. A. A. (2006). Doenças do sistema nervoso de bovinos no Brasil. In Coleção Vallée (AGNS).

Bracarense, A. P. F. L., Vidotto, O., \& Cruz, G. D. (2001). Transmissão congênita de Babesia bovis. Arquivo Brasileiro de Medicina Veterinária e Zootecnia, 53(4), 479-481. https://doi.org/10.1590/S010209352001000400017

Costa, V. M. M., Ribeiro, M. F. B., Duarte, G. A. F. P., Soares, J. F., Azevedo, S. S., Barros, A. T. M., Riet-Correa, F., \& Labruna, M. B. (2018). Incidência de Anaplasma marginale, Babesia bigemina e Babesia bovis em bezerros no semiárido paraibano. Pesquisa Veterinária Brasileira, 38(4), 605-612. https://doi.org/10.1590/1678-5150-pvb-4786

Farias, N. A., \& Lemos, R. A. A. (2007). Tristeza parasitária bovina. In Doenças de Ruminantes e Equídeos (Vol. 1, pp. 524-532). Pallotti Santa Maria.

Farias, N. A. R. (2007). Carrapato dos bovinos. In Doenças de ruminantes e equídeos (Vol. 2). Palotti Santa Maria.

Farias, Nara Amélia Rosa. (1995). Diagnóstico e controle da tristeza parasitária bovina. Livraria e Editora Agropecuária.

Gonçalves, P. M. (2000). Epidemiologia e controle da tristeza parasitária bovina na região sudeste do Brasil. Ciência Rural, 30(1), 187-194. https://doi.org/10.1590/S0103-84782000000100030

Gonçalves, R. C., Silva, A. A., Ferreira, D. O. L., Chiacchio, S. B., Lopes, R. S., Borges, A. S., \& Amorim, R. M. (2011). Tristeza Parasitária em bovinos na região de Botucatu - SP: estudo retrospectivo de 1986 - 2007. Semina: Ciências Agrárias, 32(1), 307. https://doi.org/10.5433/1679$\underline{0359.2011 \mathrm{v} 32 \mathrm{n} 1 \mathrm{p} 307}$

Kessler, R. H. (2001). Considerações sobre a transmissão de Anaplasma marginale. Pesquisa Veterinária Brasileira, 21(4), 177-179. https://doi.org/10.1590/S0100-736X2001000400009

Krolow, R. C. P. (2002). Imunidade passiva e ativa contra Babesia bovis e Babesia bigemina em terneiros nascidos na primavera em área marginal para o vetor Boophilus microplus. Dissertação de Mestrado em Medicina Veterinária. Faculdade de Veterinária. Universidade Federal de Pelotas.

Matias, J., Koller, W. W., \& Garcia, M. V. (2013). Classificação, distribuição geográfica, ciclo biológico e importância econômica das principais espécies de carrapato no Brasil. In Carrapatos no Brasil (W. W., p. 192). EMBRAPA.

Oliveira, P. A., Alves, D. M., Zamboni, R., Scheid, H. V., Alberti, T. S., Marcolongo-Pereira, C., Schild, A. L., \& Sallis, E. S. V. (2018). Babesiose cerebral em bezerros. Pesquisa Veterinária Brasileira, 38(5), 832-834. https://doi.org/10.1590/1678-5150-pvb-5424

Rodrigues, A., Rech, R. R., Barros, R. R., Fighera, R. A., \& Barros, C. S. L. (2005). Babesiose cerebral em bovinos: 20 casos. Ciência Rural, 35(1), 121-125. https://doi.org/10.1590/S0103-84782005000100019

Sacco, A. M. S. (2001). Controle/profilaxia da tristeza parasitária bovina. Embrapa Pecuária SulCircular Técnica (INFOTECA-E), 1-3.

Santarosa, B. P., Dantas, G. N., Ferreira, D. O. L., Rocha, N. S., Gonçalves, R. C., Amorim, R. M., \& Chiacchio, S. B. (2013). Infecção neurológica por Babesia bovis em bovino neonato: relato de caso. Veterinária e Zootecnia, 9-14.

Santos, L. R., Araújo, F. R., Ramos, C. A. N., Gomes, C. C. G., Gaspar, E. B., \& Benavides, M. V. (2013). Tristeza parasitária bovina: avanços no controle. In R. \& K. Andreotti (Ed.), Carrapatos no Brasil (W. W., p. 192). EMBRAPA.

Silva, J. B., Lopes, C. T. A., Pinheiro, C. P., Lima, D. H. S., Silva, R. S. L., Fonseca, A. H., Araújo, F. 
R., \& Barbosa-Neto, J. D. (2013). Prevalência sorológica e molecular de Babesia bovis e Babesia bigemina em búfalos (Bubalus bubalis) na Ilha de Marajó, Pará. Pesquisa Veterinária Brasileira, 33(7), 847-850. https://doi.org/10.1590/S0100-736X2013000700003

Siqueira, F., Cardoso, F. F., Gomes, C. C. G., Regitano, L. C. A., \& Machado, M. A. (2013). Resistência genética de bovinos ao carrapato Ripicephalus (Boophilus) microplus. In R. \& K. Andreotti (Ed.), Carrapatos no Brasil (p. 192). EMBRAPA.

Taylor, M. A., Coop, R. L., \& Wall, R. L. (2017). Parasitologia Veterinária. Guanabara Koogan.

Recebido: 4 de abril, 2020

Aprovado: 20 de maio, 2020.

Disponível online: 4 de setembro, 2020.

Licenciamento: Este artigo é publicado na modalidade Acesso Aberto sob a licença Creative Commons Atribuição 4.0 (CC-BY 4.0), a qual permite uso irrestrito, distribuição, reprodução em qualquer meio, desde que o autor e a fonte sejam devidamente creditados. 